\title{
A symptomatic pelvic rib
}

\author{
M. Podgórski ${ }^{1}$, D. Gwizdała' ${ }^{1}$, P. Flont ${ }^{2}$, K. Niedzielski², M. Polguj ${ }^{3}$, P. Grzelak ${ }^{4}$ \\ ${ }^{1}$ Department of Radiology and Imagining Diagnostic, Polish Mother's Memorial Hospital Research Institute, Lodz, Poland \\ 2Department of Orthopedics and Traumatology, Polish Mother's Memorial Hospital Research Institute, Lodz, Poland \\ ${ }^{3}$ Department of Angiology, Medical University of Lodz, Poland \\ ${ }^{4}$ Department of Radiology and Diagnostic Imaging, Medical University of Lodz, Poland
}

[Received: 8 February 2017; Accepted: 12 June 2017]

Pelvic rib is a rare anomaly where ectopic rib is found in a pelvic region. It is usually found occasionally in asymptomatic patients. We report a case of 15-year-old male, diagnosed with a symptomatic pelvic rib. It had an unusual presentation creating a pseudotumour associated with pain and reduced range of motion in the hip joint. Patient was operated on with good result and final diagnosis was confirmed in pathological examination. (Folia Morphol 2018; 77, 2: 406-408)

Key words: congenital anomaly, accessory rib, pelvic digit, ischium, eleventh finger

\section{INTRODUCTION}

The pelvic rib (pelvic digit) is a rib-like bony structure that may arise from bones of the pelvis. It is considered to be a congenital abnormality of the developing bone in close proximity to a bony pelvis [3]. It can consist from a few segments creating pseudoarticulations (also with surrounding bones). Pelvic rib usually causes no symptoms and is incidentally found on radiograms; however, it should be differentiate from other pathologies like osteochondroma or dysontogenetic tumours [6, 10].

In this report we present a management of a 15-year-old male who was operated on for a symptomatic pelvic rib that arose from an ischium. During the surgery, data from conventional X-ray, computed tomography (CT) and magnetic resonance imaging (MRI) were applied.

\section{CASE REPORT}

The 15-year-old, male presented with a 2-year history of progressive pain in the right gluteal area, radiating to the posterior part of the thigh and to the calf. Symptoms occurred periodically, initially following jumping exercise. Six to eight weeks prior to admission symptoms rapidly aggravated, limiting mobility with need for crutches, also reducing the range of motion in right hip joint. Patient had no medical history of trauma or musculoskeletal abnormalities. Initially, the X-ray of the right hip showed no abnormalities. On pelvic MRI compression of roots of the spinal cord was ruled out. Patient was admitted to Department of Orthopaedics and Traumatology. Physical examination revealed a palpable tumour, located medially to the right ischial tuberosity (on the border of buttock and upper thigh) with pain upon compression. Slightly positive Lasegue's sign was present. Basic laboratory tests (complete blood count, ESR, CRP) were within normal limits. Pelvis $\mathrm{X}$-ray revealed an accessory, well calcified linear structure projected over the ischium (Fig. 1A). Diagnosis was completed by a non-contrast CT scan confirming the presence of inhomogeneous area in the medial group of the thigh muscles, with a longitudinal bony structure and smaller band-like calcifications (Fig. 1B). The remaining bone structures in the pelvis and right thigh did not show any pathological changes. Due to unusual character of the soft tissues surround-

Address for correspondence: M. Podgórski, MD, PhD, Department of Radiology and Imagining Diagnostic, Polish Mother's Memorial Hospital Research Institute, ul. Rzgowska 281/289, 93-338 Łódź, Poland, tel: +48 422711 571, e-mail: michal.podgorski@umed.lodz.pl 

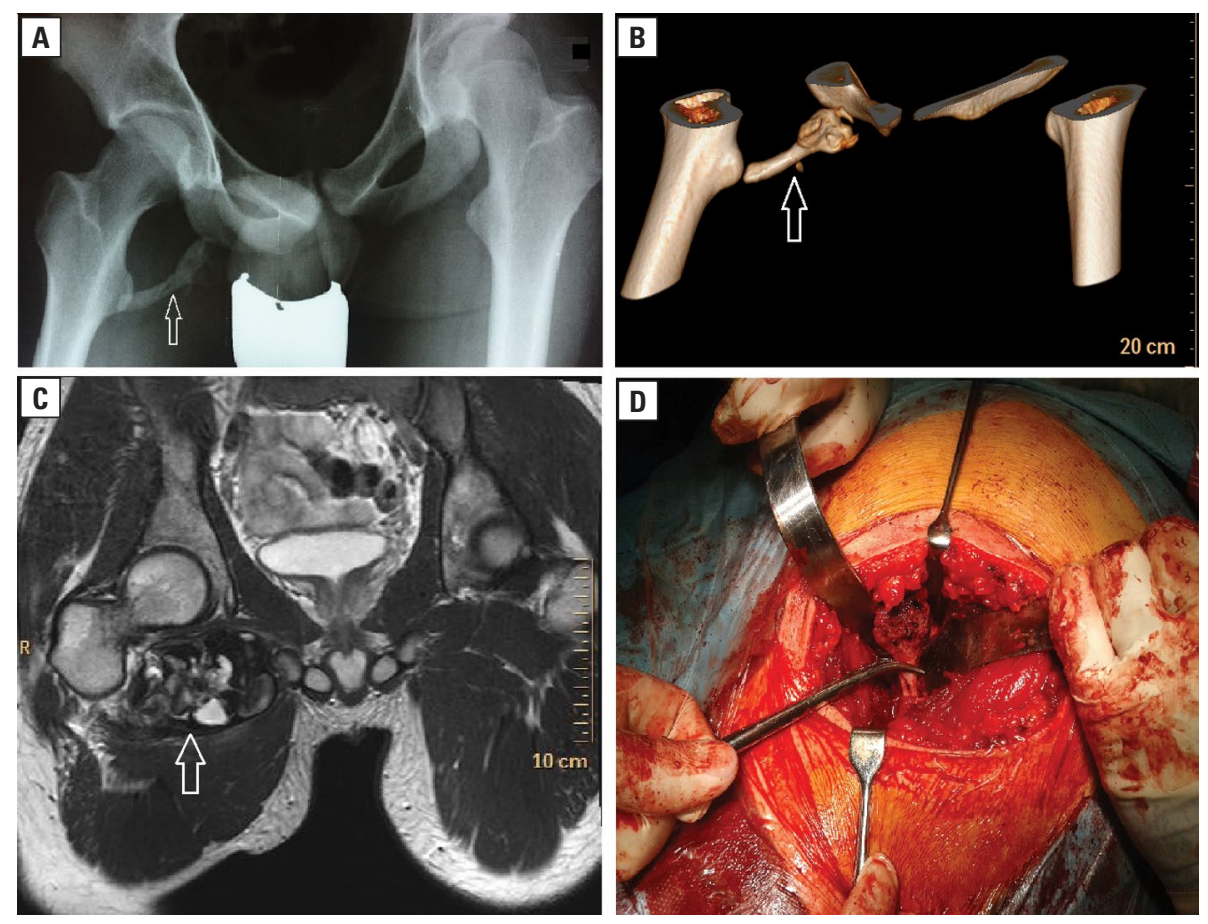

Figure 1. A. Antero-posterior pelvic X-ray with the limbs adducted. The pelvic rib (arrow) presents as a bony structure between the right ischium and lesser trochanter of the right femur. Despite the positioning asymmetry remaining bones appear normally; B. Three-dimensional volume rendering (VR) multi-detector computed tomography showing the pelvic rib (arrow), size $60 \times 20 \times 10 \mathrm{~mm}$ articulating with the ischium; C. Magnetic resonance imaging in transverse T1W sequences. Well defined, intramuscular mass (arrow) with a size of $71 \times 60$ $\times 56 \mathrm{~mm}$ and non-uniform signal intensity (containing elements of haemoglobin degradation) compressing surrounding structures;

D. Removal of the pelvic rib (held with pean).

ing the bone protuberance a two phase MRI was performed which revealed a well isolated mass $(71 \times 60 \times 56 \mathrm{~mm})$ located between head, neck, and lesser trochanter of right femur and ischium, which had a signal equivalent to a haematoma. The mass was located intramuscularly, compressing surrounding tissues and dislocating adjacent muscle groups. In its posterior part there was a longitudinal, bone-like structure $(60 \times 15 \mathrm{~mm})$. The lateral part of this mass had a typical structure of the long bone including cortical bone (Fig. 1C). The sciatic nerve was situated $10 \mathrm{~mm}$ dorsally of the tumour. The femoral artery, vein and nerve were located $35 \mathrm{~mm}$ in front of the described mass. There was no lymphadenopathy. Due to increasing pain limiting physical activity and the need to exclude malignancy the patient was qualified for surgery. The bony mass, macroscopically resembling a small rib $(6 \mathrm{~cm}$ in length and crosssection of $2 \times 1 \mathrm{~cm}$ ) and a surrounding hematoma were removed (Fig. 1D). Histopathology showed a typical bone structure. Fifteen days after surgery the patient was discharged with no deficiency in range of motion.

\section{DISCUSSION}

The majority of additional ribs are connected to transitional vertebrae of the cervical or lumbar spine [2]. Their occurrence in the pelvis is unusual. Ectopic ribs usually arise from the ilium, sacrum and coccyx [3-5]; however, an association with the ischium, like in our case, is very rare. To the best of our knowledge, only Granieri and Bacarini [4] and Greenspan and Norman [5] describe 3 patients with a similar lesion adjacent to the ischium. Pelvic ribs can develop as a single, longitudinal, well calcified structure, with a proper differentiation of cortex and medulla and may consist of a few segments creating false joints, also with bony pelvis [7].

Occurrence of pelvic rib seems to result from the migration of embryonic mesoderm, with ability to form a rib, into the pelvic region [1]. Other authors suggest that this mass is formed during mesenchymal bone growth that occurs before the sixth week of 
foetal development [1]. However, it does not explain the occurrence of a mass in the pelvis (coccyx area, pelvis and lower abdomen).

Pelvic rib is usually found incidentally on radiograms in asymptomatic patients or may be palpated as a pseudotumour, like in the presented case. A similar clinical presentation can be observed after bone trauma (avulsion fractures of the pelvis, subperiosteal haematomas), in nail-patella syndrome, osteochondroma, fibrodysplasia ossificans progressiva or in some dysontogenetic tumours (i.e. teratomas) [3-5]. Therefore the identification of this pathology as a developmental anomaly is important. In doubtful cases $\mathrm{CT}$ is the best diagnostic method of choice and MRI can provide additional information on changes in the surrounding soft tissues. However, this case indicates that changes observed in MRI can be misleading.

Surgery is reserved to symptomatic cases. Our report is the third description of a complete removal of such a lesion. The pain in our patient subsided and full range of motion of the hip joint was restored $[8,9]$.

\section{CONCLUSIONS}

Pelvic rib can be symptomatic with pain and reduced range of motion in the hip joint. Diagnostic workup, including MRI, may over-interpret the reaction of surrounding soft tissues and falsely suggest a malignant process.

\section{REFERENCES}

1. Casey MC, Phancao JP, Pressacco J. Answer to case of the month \# 106. Pelvic Digit. Can Assoc Radiol J. 2006; 57(1): 51-53, indexed in Pubmed: 16719214.

2. Erken E, Ozer HTE, Gulek B, et al. The association between cervical rib and sacralization. Spine (Phila Pa 1976). 2002; 27(15): 1659-1664, indexed in Pubmed: 12163729.

3. Goyen M, Barkhausen J, Markschies NA, et al. The pelvic digit--a rare developmental anomaly. A case report with CT correlation and review of the literature. Acta Radiol. 2000; 41(4): 317-319, indexed in Pubmed: 10937749.

4. Granieri GF, Bacarini L. The pelvic digit: five new examples of an unusual anomaly. Skeletal Radiol. 1996; 25(8): 723-726, indexed in Pubmed: 8958617.

5. Greenspan A, Norman A. The "pelvic digit" - an unusual developmental anomaly. Skeletal Radiol. 1982; 9(2): 118-122, indexed in Pubmed: 7163821.

6. Lame E. Case report 32. Skeletal Radiology. 1997; 2(1): 47-48, doi: 10.1007/bf00364629.

7. Maegele M. Pelvic digit as a rare cause of chronic hip pain and functional impairment: a case report and review of the literature. J Med Case Rep. 2009; 3: 139, doi: 10.1186/17521947-3-139, indexed in Pubmed: 20062776.

8. McGlone BS, Hamilton S, FitzGerald MJ. Pelvic digit: an uncommon developmental anomaly. Eur Radiol. 2000; 10(1): 89-91, doi: 10.1007/s003300050010, indexed in Pubmed: 10663721.

9. Nguyen VD, Matthes JD, Wunderlich CC. The pelvic digit: $\mathrm{CT}$ correlation and review of the literature. Comput Med Imaging Graph. 1990; 14(2): 127-131, indexed in Pubmed: 2110499.

10. Pandey V, Thakur AS, Acharya KKv, et al. The pelvic digit "eleventh finger". Indian J Orthop. 2009; 43(1): 97-98, doi: 10.4103/0019-5413.45332, indexed in Pubmed: 19753190. 